\section{(6) OPEN ACCESS}

\title{
Improving the healthcare response to domestic violence and abuse in sexual health clinics: feasibility study of a training, support and referral intervention
}

\author{
Alex Hardip Sohal, ${ }^{1}$ Neha Pathak, ${ }^{2}$ Sarah Blake, ${ }^{3}$ Vanessa Apea, ${ }^{4}$ Judith Berry, ${ }^{5}$

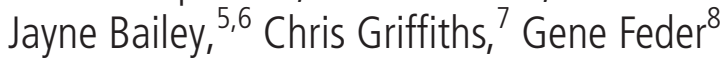

${ }^{1}$ Queen Mary University of London, Centre for Primary Care and Public Health, Barts and The London School of Medicine and Dentistry, London, UK

'Women's Health Research Unit, Queen Mary University of London, University of Bristol School of Social and Community Medicine, Bristol, UK ${ }^{3}$ University of Bristol, School of Social and Community Medicine Bristol, UK

${ }^{4}$ Barts Health NHS Trust, Sexual Health, London, UK

${ }^{5}$ University Hospitals Bristol NHS Foundation Trust, Bristol Sexual Health Centre, Bristol, UK

${ }^{6}$ University of Bristol, School of Social and Community Medicine, Bristol, UK

${ }^{7}$ Queen Mary University of London, Centre for Primary Care and Public Health, London, UK ${ }^{8}$ University of Bristol, Centre for Academic Primary Care, Bristol, UK

\section{Correspondence to} Dr Alex Hardip Sohal, Queen Mary University of London, Centre for Primary Care and Public Health, Barts and The London School of Medicine and Dentistry, Yvonne Carter Building, 58 Turner Street, London E1 2AB, UK; ahsohal@ yahoo.co.uk

Received 21 September 2016 Revised 27 February 2017 Accepted 16 March 2017 Published Online First 19 July 2017

\section{SLinked}

- http://dx.doi.org/10.1136/ sextrans-2017-053322

- http://dx.doi.org/10.1136/ sextrans-2017-053411

\section{Check for updates}

To cite: Sohal $\mathrm{AH}$

Pathak N, Blake $S$,

et al. Sex Transm Infect

2018:94:83-87.

\section{ABSTRACT}

Objectives Sexual health and gynaecological problems are the most consistent and largest physical health differences between abused and non-abused female populations. Sexual health services are well placed to identify and support patients experiencing domestic violence and abuse (DVA). Most sexual health professionals have had minimal DVA training despite English National Institute for Health and Care Excellence recommendations. We sought to determine the feasibility of an evidence-based complex DVA training intervention in female sexual health walk-in services (IRIS ADViSE: Identification and Referral to Improve Safety whilst Assessing Domestic Violence in Sexual Health Environments).

Methods An adaptive mixed method pilot study in the female walk-in service of two sexual health clinics. Following implementation and evaluation at site 1, the intervention was refined before implementation at site 2. The intervention comprised electronic prompts, multidisciplinary training sessions, clinic materials and simple referral pathways to IRIS ADViSE advocateeducators (AEs). The pilot lasted 7 weeks at site 1 and 12 weeks at site 2. Feasibility outcomes were to assign a supportive DVA clinical lead, an IRIS ADViSE AE employed by a local DVA service provider, adapt electronic records, develop local referral pathways, assess whether enquiry, identification and referral rates were measurable.

Results Both sites achieved all feasibility outcomes: appointing a supportive DVA clinical lead and IRIS ADViSE AE, establishing links with a local DVA provider, adapting electronic records, developing local referral pathways and rates of enquiry, identification and referral were found to be measurable. Site 1: 10\% enquiry rate $(n=267), 4 \%$ identification rate $(n=16)$ and eight AE referrals. Site 2: $61 \%$ enquiry rate $(n=1090)$, a $7 \%$ identification rate $(n=79)$ and eight $A E$ referrals. Conclusions IRIS ADViSE can be successfully developed and implemented in sexual health clinics. It fulfils the unmet need for DVA training. Longer-term evaluation is recommended.

\section{INTRODUCTION}

Domestic violence and abuse (DVA) is a major public health and clinical problem affecting individuals, families, communities and society. The UK intergovernmental definition of DVA is any incident or pattern of controlling, coercive or threatening behaviour, violence or abuse between people aged $\geq 16$ years who are or have been intimate partners or family members, regardless of gender or sexuality. ${ }^{1}$ DVA can encompass, but is not limited to, psychological, physical, sexual, financial or emotional abuse. The estimated annual cost of DVA to the British National Health Service is $£ 1.7$ billion per year, additional mental health costs $£ 176$ million $^{2}$ and the aggregate UK cost including lost economic output, social services, medical and emotional costs an estimated $£ 11$ billion. ${ }^{3}$ Although DVA affects men and women, the prevalence of all DVA is higher among women and increasing since 2008/2009. ${ }^{4}$ Women experience more severe and repeated physical abuse, much more sexual abuse and more coercive control than men. ${ }^{1}$

Gynaecological and sexual health problems are the most prevalent and persistent physical health consequence of DVA. Presentations include STIs, painful sex, chronic pelvic pain, vaginal bleeding and recurrent urinary tract infections. Risk of these problems is threefold higher in abused women, and increases in a dose-response fashion with coexisting sexual and physical abuse. ${ }^{5}$ DVA is also associated with an increased risk of unintended pregnancy, induced abortion, increased sexual risk taking and inconsistent condom use. ${ }^{67}$ Lifetime prevalence of DVA in women attending sexual health services (47\%) is higher than in the general population. ${ }^{8}$

Sexual health services can be the first point of contact for women who have experienced DVA, so is an appropriate setting in which to identify and support women affected by DVA. ${ }^{9}$ They were listed as a setting in which all patients should be asked about DVA as a 'routine part of good clinical practice' by the National Institute for Health and Care Excellence, ${ }^{10}$ which develops evidence-based guidance for healthcare in England. However, most sexual health professionals have had minimal training in identifying and responding to DVA.

IRIS (Identification and Referral to Improve Safety) is an evidence-based complex intervention including DVA training, support and referrals to specialist DVA advocate-educators (AE) in primary care, designed to improve the healthcare response to women affected by DVA. A cluster randomised controlled trial in Hackney and Bristol general practices showed that IRIS increased the identification of women affected by DVA (currently or 
historically) 3-fold, discussion about referral 22-fold and actual referral 6-fold. ${ }^{11}$ The IRIS intervention is likely to be cost-effective $^{12}$ as well as acceptable to service users ${ }^{13}$ and professionals. ${ }^{14}$ The IRIS model has been endorsed by the Royal College of General Practitioners and is frequently cited in guidance as good practice. $^{10}{ }^{15-17}$ IRIS has been commissioned in 34 localities, involving $>1000$ UK general practices.

\section{Aim}

Determining the feasibility of developing and implementing an IRIS-type model in sexual health services: IRIS ADViSE (Assessing Domestic Violence in Sexual Health Environments).

\section{METHODS}

Design

We used an adaptive pilot study design ${ }^{18}$ where the experience and data at site 1, in East London, led to modifications at site 2, in Bristol. We used a mixed methods approach to evaluation. This comprised monitoring of predefined feasibility outcomes which included analysis of numerical data extracted from the electronic medical record. Qualitative analysis of staff interviews is reported in a separate paper. ${ }^{19}$

\section{Setting and participants}

Both sites were female walk-in sexual health services. Site 1 was an east London clinic, serving an inner-city multiethnic population. Site 2 was a Bristol clinic, serving an urban population. Both clinics saw commuters and those referred from primary care, community and regional clinics.

\section{Process, intervention and procedures}

Figure 1 summarises the core methodology including the IRIS ADViSE intervention details. Sexual health-based case studies integrating best practice in the healthcare response to DVA with routine sexual healthcare were included. Local steering groups facilitated discussion on how best to develop and implement IRIS ADViSE locally. At site 1, adaptation of electronic records and paper triage forms occurred simultaneously; while at site 2, a question on abuse was added to the paper triage form, 3 months prior to the abuse questions' integration into the electronic records. Patients on arrival at reception complete the paper triage form that contains questions about reason for attendance.

Table 1 summarises how site 1 informed implementation at site 2 . At site 1, it was not mandatory for staff to indicate whether they had asked about DVA whereas it was at site 2 . This difference was instituted directly due to the results from site 1 .

\section{Ethical aspects}

We sought the views of chairs of local ethics committees at both sites. We received written confirmation that the collection of data from the medical record constituted routine service evaluation.

\section{Outcome measures and data collection}

The predefined feasibility outcome measures were whether

- a DVA clinical lead was appointed in each sexual health service

- a local DVA services' provider collaborated with the sexual health service on this project

- an advocate educator was appointed

- an electronic pro forma included assessment of DVA

- local referral pathways were developed

- enquiry, identification and referral rates were measurable. Handwritten notes were kept on the progress of the project by AS at site 1 and NP at site 2. Anonymised aggregated data, following the first session of training, once the pilot project was live was collected for 7 weeks at site 1 and 12 weeks at site 2 , on the number of women who

- attended the walk-in sexual health service at east London and Bristol

- were asked about DVA by staff

- were identified as being affected by DVA, either currently or historically

- were referred to the AE.

We aimed to collect 3 months of baseline data at each site.

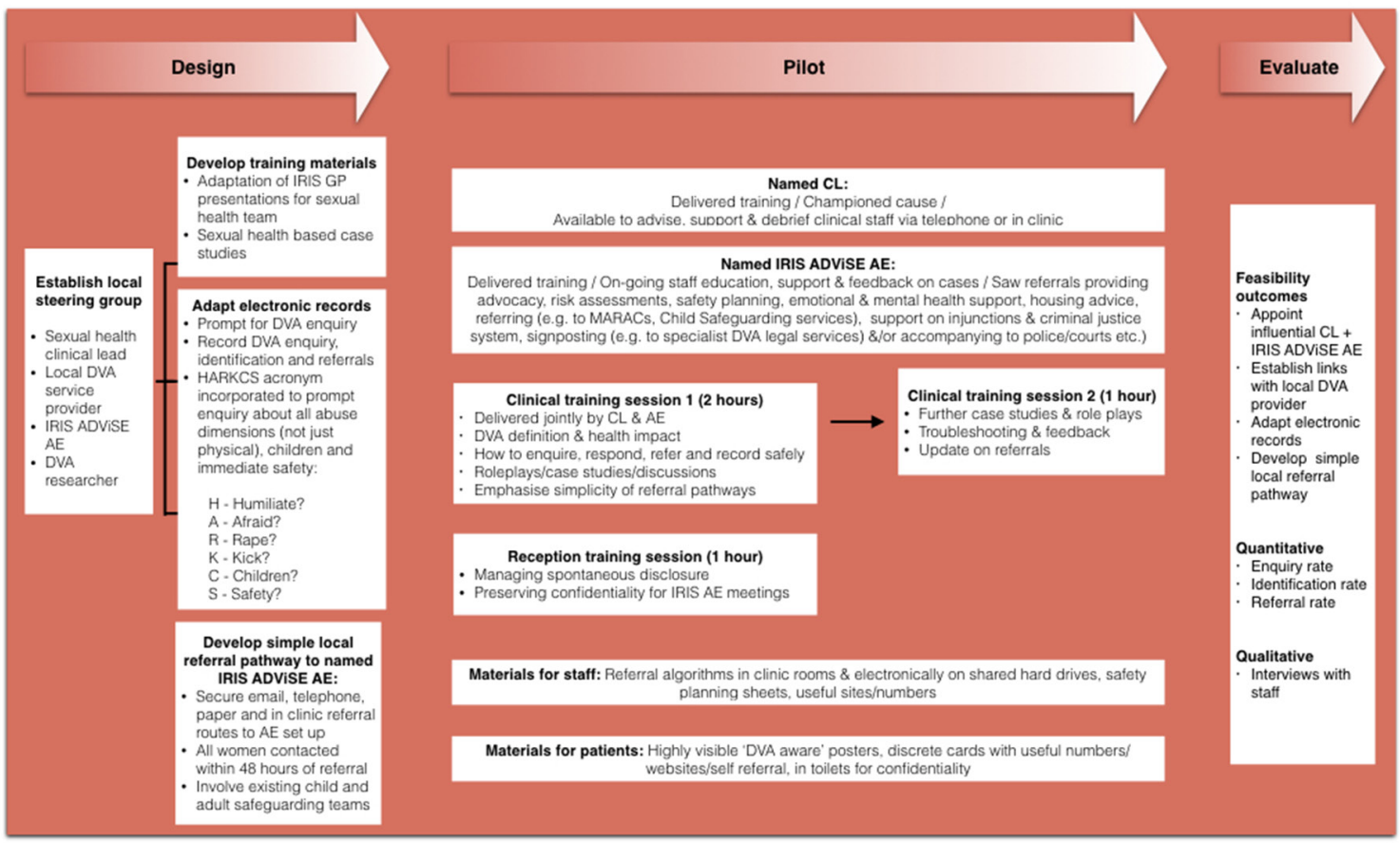

Figure 1 IRIS ADViSE adaptive pilot study: core methodology. 


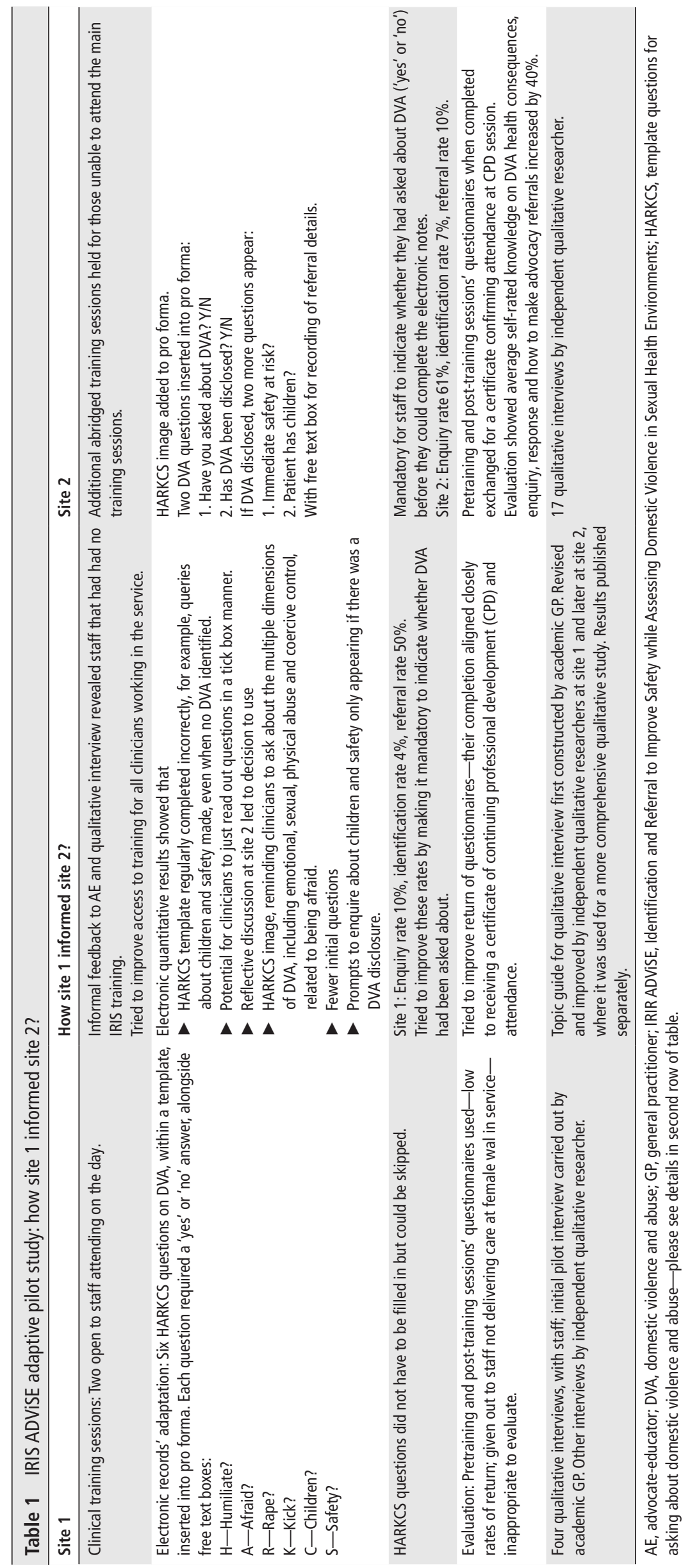




\section{RESULTS}

At both sites, all feasibility outcomes were achieved, in that process measures (appointing key staff; see below) were attained and potential future outcome measures (enquiry, identification and referral rates) were measurable.

\section{Process measures attained}

Local sexual health consultants were appointed as DVA CLs, local DVA service providers collaborated on the project and AEs, experienced in advocacy and training, were successfully recruited. DVA enquiry was integrated into the standard electronic template, reminding sexual health staff to ask about DVA. Adapted IRIS training was delivered to the sexual health team in their weekly continuing professional development education session. Clear local referral pathways on how best to contact the IRIS AE were established.

\section{Outcome measures measurable}

At site 1 , over 7 weeks, the DVA enquiry rate was 10\% with 267 women out of 2568 women attending asked about DVA. The DVA identification rate was $4 \%$ with 12 out of the 267 women asked about abuse, affected by abuse. Four additional cases of DVA were identified via the self-triage form. Overall 50\% (8 out of 16) of the women affected by abuse were referred to the AE.

At site 2, over 12 weeks, the DVA enquiry rate was $61 \%$ with 1090 women out of 1775 women attending asked about DVA. The DVA identification rate was $7 \%$ with 79 out of the 1090 women asked about abuse affected by abuse. At site 2, 13 of the 79 cases of DVA were identified via the self-triage form. Overall $10 \%$ ( 8 out of 79 ) of the women affected by abuse were referred to the AE.

During the 3 months preceding the start of each pilot project, there were no electronically recorded cases of DVA identified and no referrals to DVA specialist services. At site 2, during this 3 -month period, it is unknown how many women disclosed abuse on the paper self-triage form but none were referred to specialist services.

\section{DISCUSSION}

This adaptive pilot study shows that it is feasible to develop and implement an IRIS-based DVA training and referral package for sexual health clinics. The intervention resulted in the identification and referral of women affected by DVA, suggesting that it is a potentially effective intervention. It is also feasible to collect data for quantitative evaluation of the intervention's impact on DVA enquiry, identification and referral for advocacy.

The actual rates of DVA enquiry, identification and referral, all show a change from baseline which means that IRIS ADViSE affects clinician behaviour. The limitations of this study is that it is not powered to estimate the size of this effect, nor can it conclusively exclude a secular trend due to the non-randomised study design.

This study's strength was its adaptive design, allowing transparent, sequential, rapid refinement of IRIS ADViSE. Piloting IRIS ADViSE in more than one site acknowledges variation in sexual health service delivery and confirms the feasibility of tailoring the intervention to individual services.

Comparison of sites 1 and 2 shows that the DVA enquiry rate increased over fivefold, from $10 \%$ to $61 \%$ with just under a doubling in the DVA identification from $4 \%$ to $7 \%$ and a drop in referral rates from $50 \%$ at site $1 \%$ to $10 \%$ at site 2 , meaning that the absolute numbers of women referred to an $\mathrm{AE}$ at the two sites was the same (eight women at each site). These differences are most likely to be due to the adaptive change made at site 2, making it mandatory for clinicians to indicate whether DVA had been asked about before proceeding.

As IRIS ADViSE is introduced into diverse sexual health services and settings, the local steering groups should consider the cons and pros to each approach. For example, making it mandatory to record whether DVA enquiry has occurred appears to be associated with a drop in the referral rates through higher enquiry and identification rates. IRIS ADViSE may benefit women affected by DVA who are asked about DVA but decide to not disclose (large numbers as estimated by DVA survey prevalence figures) and may benefit women identified as being affected by DVA who are offered referral but decide that they do not want to be referred.

A recent DVA screening study, in multiple hospital settings, found that in genitourinary medicine $5.7 \%$ patients screened reported ever experiencing DVA, using a training intervention delivered by a local specialist DVA provider, ${ }^{20}$ without clinician-delivered clinically relevant training, nor using validated DVA questions like HARK, ${ }^{21}$ nor reporting how abuse questions were integrated into the medical record. This intervention's DVA enquiry rate (ie, the number of patients asked about DVA) and referral rate for advocacy are unknown (ie, source of referrals received by the hospital-based domestic violence advisor undetermined). To allow a quantitative comparison with IRIS ADViSE requires the latter's identification rates to be adjusted so that the denominator is changed from the total number asked about DVA to the total number who attended the service. At site 1 , this adjusted identification rate is $0.6 \%(=16 / 2568)$; at site 2 this is $4.5 \%(=79 / 1775)$, which is a similar level to the Warren-Gash et al study. These figures support and we recommend retaining site 2's mandatory recording of whether DVA enquiry has occurred.

In summary, the IRIS intervention has been successfully adapted for female walk-in sexual health services. Further evaluation of IRIS ADViSE with appropriate refinement for each setting is now required to confirm its effectiveness prior to national scaling up. A quasi-experimental approach would enable a pragmatic phased implementation of IRIS ADViSE to other sexual health clinics, including HIV clinics, pregnancy advisory services, outreach services, psychosexual clinics, male and LGBT services. Future studies, using this adaptive, novel approach, should extend DVA enquiry ensuring a competent and compassionate clinical response whenever abuse is identified.

Contributors AHS designed the pilot evaluation in site 1 and was responsible for the study at that site. She drafted and revised the manuscript. NP designed the pilot evaluation in site 2 and was responsible for the study at that site. She contributed to manuscript revision and constructed the figures and tables. GF contributed to the design of the studies and contributed to manuscript revision. SB, VA, JB, JB and CG contributed to manuscript revision. SB was also an advocate educator at site 2. VA and JB were responsible for service delivery at sites 1 and 2, respectively, assisting in anonymous data collection.

Funding Should read: "AHS and NP were funded by the National Institute for Health Research (NIHR) for their academic clinical lectureship and academic clinical fellowship respectively, during the preparation of this manuscript. NP was funded by the Wellcome Trust during the implementation of the pilot. The research and AHS was supported by the NIHR Collaboration for Leadership in Applied Health Research and Care (CLAHRC) North Thames at the Barts Health NHS Trust; and the West CLAHRC. The views expressed are those of the authors and not necessarily those of the NHS, the NIHR or the Department of Health. Tower Hamlets Public Health provided funding for work carried out at site 1. GF is a consultant for the IRIS programme for which the University of Bristol receives a fee. None of these funding organisations had any role in the design, implementation, and interpretation nor reporting of this work."

Competing interests None declared. 
Provenance and peer review Not commissioned; externally peer reviewed.

Data sharing statement Additional unpublished data are available from the corresponding author by contacting via email.

Open Access This is an Open Access article distributed in accordance with the terms of the Creative Commons Attribution (CC BY 4.0) license, which permits others to distribute, remix, adapt and build upon this work, for commercial use, provided the original work is properly cited. See: http://creativecommons.org/licenses/by/4.0/

(C) Article author(s) (or their employer(s) unless otherwise stated in the text of the article) 2018. All rights reserved. No commercial use is permitted unless otherwise expressly granted.

\section{REFERENCES}

1 Satistics OfN. C. Chapter 4: Violent Crime and Sexual Offences - Intimate Personal Violence and Serious Sexual Assault. Crime Statistics, Focus on Violent Crime and Sexual Offences 2013/14 Release, 2015.

2 Walby S. The cost of domestic violence: up-date 2009. Lancaster University, 2009.

3 Walby S, Olive P. Estimating the costs of gender-based violence in the European Union: European Institute for Gender Equality, 2014

4 Walby S, Towers J, Francis B. Is violent crime increasing or decreasing? A new methodology to measure repeat attacks making visible the significance of gender and domestic relations. Br J Criminol 2016;56:1203-34.

5 Campbell JC. Health consequences of intimate partner violence. Lancet 2002;359:1331-6.

6 Miller E, McCauley HL, Tancredi DJ, et al. Recent reproductive coercion and unintended pregnancy among female family planning clients. Contraception 2014:89:122-8.

7 Hall M, Chappell LC, Parnell BL, et al. Associations between intimate partner violence and termination of pregnancy: a systematic review and meta-analysis. PLoS Med 2014;11:e1001581.

8 Loke WC, Torres C, Bacchus L, et al. Domestic violence in a genitourinary medicine setting-an anonymous prevalence study in women. Int J STD AIDS 2008;19:747-51.
9 Bacchus LJ, Bewley S, Vitolas CT, et al. Evaluation of a domestic violence intervention in the maternity and sexual health services of a UK hospital. Reprod Health Matters 2010;18:147-57.

10 National Institute for Health and Care Excellence. Domestic violence and abuse: multiagency working NICE guideline. 2014.

11 Feder G, Davies RA, Baird K, et al. Identification and referral to improve safety (IRIS) of women experiencing domestic violence with a primary care training and support programme: a cluster randomised controlled trial. Lancet 2011;378:1788-95.

12 Devine A, Spencer A, Eldridge $S$, et al. Cost-effectiveness of identification and referral to improve safety (IRIS), a domestic violence training and support programme for primary care: a modelling study based on a randomised controlled trial. BMJ Open 2012:2:e001008.

13 Malpass A, Sales K, Johnson M, et al. Women's experiences of referral to a domestic violence advocate in UK primary care settings: a service-user collaborative study. $\mathrm{Br} J$ Gen Pract 2014;64:e151-8.

14 Yeung $\mathrm{H}$, Chowdhury N, Malpass A, et al. Responding to domestic violence in general practice: a qualitative study on perceptions and experiences. Int J FamilyMed 2012;2012:1-7.

15 WHO. Responding to intimate partner violence and sexual violence against women: who clinical and policy guidelines. 2013.

16 Domestic Homicide Review: lessons learnt. Home Office 2013.

17 HM Government: Ending violence against women and girls Strategy 2016 - 2020: London, 2016.

18 Kairalla JA, Coffey CS, Thomann MA, et al. Adaptive trial designs: a review of barriers and opportunities. Trials 2012;13:145

19 Morden A, Bailey J, Pathak N, et al. Assessing for domestic violence in Sexual Health Environments: a qualitative study.

20 Warren-Gash C, Bartley A, Bayly J, et al. Outcomes of domestic violence screening at an acute London trust: are there missed opportunities for intervention? BMJ Open 2016;6:e009069.

21 Sohal H, Eldridge S, Feder G. The sensitivity and specificity of four questions (HARK) to identify intimate partner violence: a diagnostic accuracy study in general practice. BMC Fam Pract 2007;8:49. 